\title{
Reversible conduction block in peripheral nerve using electrical waveforms
}

\author{
Niloy Bhadra1', Tina L Vrabec², Narendra Bhadra² \& Kevin L Kilgore ${ }^{*}, 3,4$ \\ ${ }^{1}$ Department of Physical Medicine \& Rehabilitation, MetroHealth Medical Center, Cleveland, OH 44109, USA \\ ${ }^{2}$ Department of Biomedical Engineering, Case Western Reserve University, Cleveland, $\mathrm{OH} 44106$, USA \\ ${ }^{3}$ Department of Orthopaedics, MetroHealth Medical Center \& Case Western Reserve University, Cleveland, OH 44109, USA \\ ${ }^{4}$ Research Service, Louis Stokes Cleveland Department of Veterans Affairs Medical Center, Cleveland, OH 44106, USA \\ * Author for correspondence: klk4@case.edu
}

Introduction: Electrical nerve block uses electrical waveforms to block action potential propagation. Materials \& Methods: Two key features that distinguish electrical nerve block from other nonelectrical means of nerve block: block occurs instantly, typically within $1 \mathrm{~s}$; and block is fully and rapidly reversible (within seconds). Results: Approaches for achieving electrical nerve block are reviewed, including kilohertz frequency alternating current and charge-balanced polarizing current. We conclude with a discussion of the future directions of electrical nerve block. Conclusion: Electrical nerve block is an emerging technique that has many significant advantages over other methods of nerve block. This field is still in its infancy, but a significant expansion in the clinical application of this technique is expected in the coming years.

First draft submitted: 22 August 2017; Accepted for publication: 15 November 2017; Published online: 14 December 2017

Keywords: action potential $\bullet$ direct current $\bullet$ electrical nerve block $\bullet$ kilohertz frequency $\bullet$ neuromodulation $\bullet$ neuroprosthesis

Electrical control of the nervous system has been an established tool in medicine since the discovery of electricity itself [1]. A single electrical pulse can produce an action potential in a neuron that, once it propagates, is indistinguishable from a normal, physiologically generated action potential, making electrical stimulation a very powerful means of controlling the nervous system within the body. However, until recently, electrical control of the nervous system was only used to activate a nerve; there was no reliable electrical means to inactivate a nerve. In this review we present what is now known about the new field of electrical nerve block, in which electrical waveforms are utilized to block or inhibit, action potential propagation. This new field opens up unique applications in bioelectronics, applications where both the upregulation and downregulation of neural activity is critical.

It should be noted that electrical nerve block, as presented in this review, is distinct from bioelectronics approaches that involve neuromodulation, which are sometimes described as 'blocking' neural activity. For example, spinal cord stimulators are loosely described as 'blocking' pain transmission. However, the direct action of spinal cord stimulators is activation of neural structures in or near the spinal cord. The resulting pain relief occurs through indirect means, possibly through the activation of inhibitory neurons within the spinal cord [2]. For the purposes of clarification, we define an electrical nerve blocking technique as one in which an action potential, traveling along a neuron, is extinguished or otherwise prevented from propagating by the application of an electrical waveform. A typical experimental setup for in vivo study of electrical nerve block is shown in Figure 1. This design, based on action potential conduction in a peripheral nerve, allows direct and conclusive measurement of nerve conduction block in real time.

Electrical nerve block has two key features that distinguish it from other nonelectrical means of nerve block. First, electrical nerve block occurs instantly, often on an action potential-by-action potential basis. Methods of electrical nerve block, as reviewed in this article, typically block nerve conduction in less than $2 \mathrm{~s}$ [3]. Methods such as kilohertz frequency alternating current (KHFAC) block have been shown to occur in less than $10 \mathrm{~ms}$ [4]. This is much faster than a pharmacologically induced block of nerve conduction, which typically requires several minutes to become fully effective. 


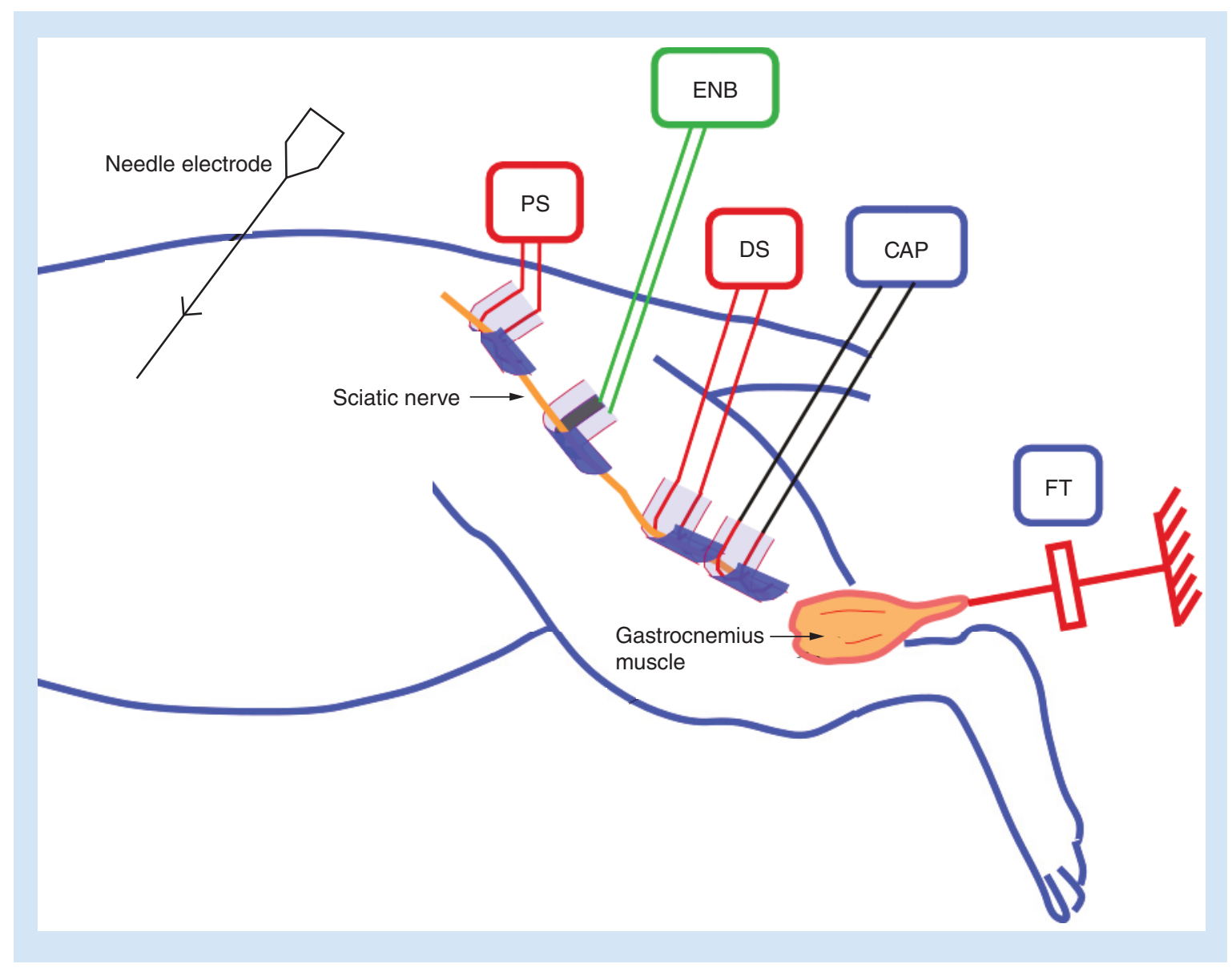

Figure 1. Typical in vivo setup for testing electrical nerve block. The blocking electrode is placed between two stimulating electrodes on the rat sciatic nerve. Compound action potentials or force from the gastrocnemius muscle are measured to evaluate conduction block in the nerve. Distal stimulation is used to verify the physical location of block and assess the conduction properties of the nerve.

CAP: Compound action potential; DS: Distal stimulation; ENB: Electrical nerve block; FT: Force transducer; PS: Proximal stimulation.

The second key feature of electrical nerve block is perhaps the most intriguing with respect to many bioelectronics applications: electrical nerve block is fully and rapidly reversible. In fact, under many conditions, electrical nerve block can be reversed within a few hundred milliseconds [3]. This provides the possibility of real-time control of neural activity, in which action potentials are alternatively either blocked or allowed to pass, based on the desired clinical effect.

We review the current status of research in this area. Fundamentally, two approaches to electrical nerve block have emerged. One approach uses an alternating current waveform with a frequency well above the normal physiological firing frequency of a nerve (KHFAC). The second approach uses direct current (DC) or modified DC (chargebalanced polarizing current [CBPC]) to produce nerve block. These approaches are reviewed, along with methods that combine the two types of waveforms to gain improved nerve block features. We also consider other electrical approaches to modulating neural activity, which are often confused with electrical nerve block in the literature. Finally, we conclude with a discussion of the future directions of electrical nerve block research and the potential clinical applications to which it may be applied.

\section{Electrical nerve block using KHFAC}

To date, the most widely studied method of reversible electrical nerve conduction block is the use of KHFAC. KHFAC nerve conduction block was first described in 1935 [5,6] but there were few papers in the 20th century, with three key publications: the first showed a gradable KHFAC block of frog sciatic nerve [7]; the second confirmed 
Figure 2. Kilohertz frequency alternating current block of the rat sciatic nerve with a sinusoidal waveform at $30 \mathrm{KHz}$. Supramaximal stimulation proximal to the block site is applied to produce gastrocnemius muscle contractions generated at $0.5 \mathrm{~Hz}$, as indicated by the red arrows. KHFAC block is delivered starting at $7 \mathrm{~s}$ (green up arrow) and is turned off $30 \mathrm{~s}$ later (green down arrow). There is an initial onset response when the KHFAC begins. Immediately after the cessation of KHFAC, gastrocnemius twitch height returns to preblock levels. KHFAC: Kilohertz frequency alternating current.

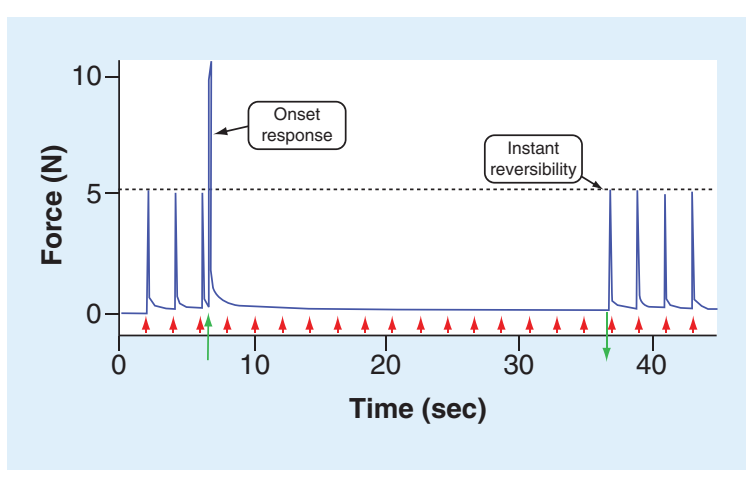

the findings of Tanner [8]; and the third was a comprehensive study of KHFAC nerve block [9]. A historical review is available in [10]. From 2004, a number of researchers began publishing investigations on KHFAC block [11-13] and initiated a systematic analysis of KHFAC block. There is now great interest in KHFAC block of motor, sensory and autonomic nerves [14]. KHFAC conduction block is being evaluated preclinically for applications in cardiac failure [15].

KHFAC nerve conduction block has some characteristic and unique features, as outlined in the following summary.

- KHFAC block is generally applied through encircling nerve cuff electrodes and produces a localized block of the underlying nerve $[3,16]$ that does not affect the neuromuscular junction;

- KHFAC block is nearly instantaneous, as shown in Figure 2. A recent study [4] used a 'counted cycles' method to measure the time to achieve complete motor block and found that it could be as rapid as 7.5-14 ms;

- KHFAC block shows rapid reversibility (Figure 2) when the KHFAC is terminated [3]. Experiments demonstrate that the nerve completely recovers within $1 \mathrm{~s}$ of the cessation of the KHFAC;

- KHFAC produces a variety of nerve responses, as a function of the frequency, amplitude and duration of delivery. These effects are outlined in Figure 3 and compared with standard electrical stimulation paradigms. These responses are complex and this has led to confusion with respect to nerve block, as discussed later in this review;

- Under certain conditions, KHFAC block produces a 'carry-over' block effect (COBE). Even after the KHFAC is stopped, there is a continuation of the depression in nerve conduction. Waataja et al. [17] showed this effect after only $1 \mathrm{~min}$ of KHFAC delivery in the vagal nerve of the rat. This is in contrast to experiments in the rat and cat sciatic nerves, where immediate reversibility can be maintained for at least $40 \mathrm{~min}$ [18]. Full nerve conduction always returns over durations of seconds-hours. An example of the carry-over effect during KHFAC block in the cat peroneal nerve is shown in Figure 4 [19]. Bhadra et al. [18] identified three distinct recovery categories from the carry-over block effect;

- KHFAC block can be modulated and the percentage of nerve that is blocked can be adjusted by changing the waveform amplitude [3]. This is in contrast to any other modality of nerve block. Modulation could be extremely useful in the clinical setting, for example, it is frequently desirable to partially block muscle activity, but retain some voluntary muscle function;

- One drawback to widespread clinical applications of KHFAC nerve block is that KHFAC produces an 'onset response' [3], which is a short duration increase in nerve activation when the KHFAC is initiated. The onset response can be a large 'twitch' response, or it can be a period of sustained activity. The motor onset response has two phases [3,20]. The first phase consists of a summated muscle twitch. This has been defined as the "Phase I onset" and it is always present. The 'Phase II onset' was defined as a period of repetitive firing that follows directly after Phase I and ends with complete or partial block. The Phase II onset can be reduced or eliminated by electrode and waveform optimization [21,22]. The onset response is an obstacle to many prospective clinical uses of KHFAC block. Therefore, research is ongoing to investigate methods to mitigate the onset response (see section on combined waveforms below); 

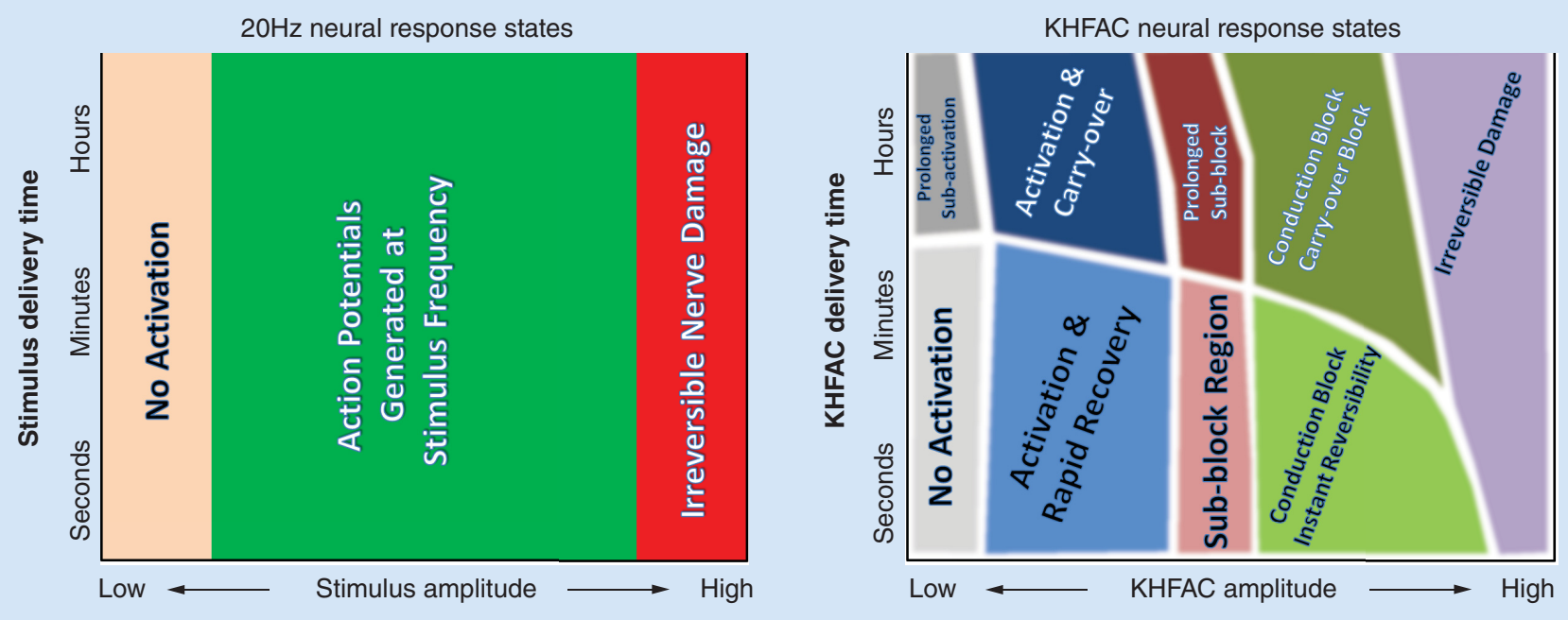

Figure 3. The effect of standard stimulation ( $20 \mathrm{~Hz}$ used as an example) compared with the effect of kilohertz frequency alternating current on nerves. This comparison focuses on the waveform amplitude versus the duration of the waveform delivery space. Within the amplitude-duration space, $20 \mathrm{~Hz}$ has three known effects on the nerve: no action; activation (at an identifiable threshold); and damage. By contrast, in this same parameter space, KHFAC has at least nine possible 'effect states', including activation, block and many longer-term effects. Note that many of these possible effect states are theoretical and have not been experimentally demonstrated. Many of the exact borders and transitions between these effect states are also, as yet, unexplored, with the exception of the transition into the conduction block region. Future research may also reveal additional effects that are currently unknown.

KHFAC: Kilohertz frequency alternating current.

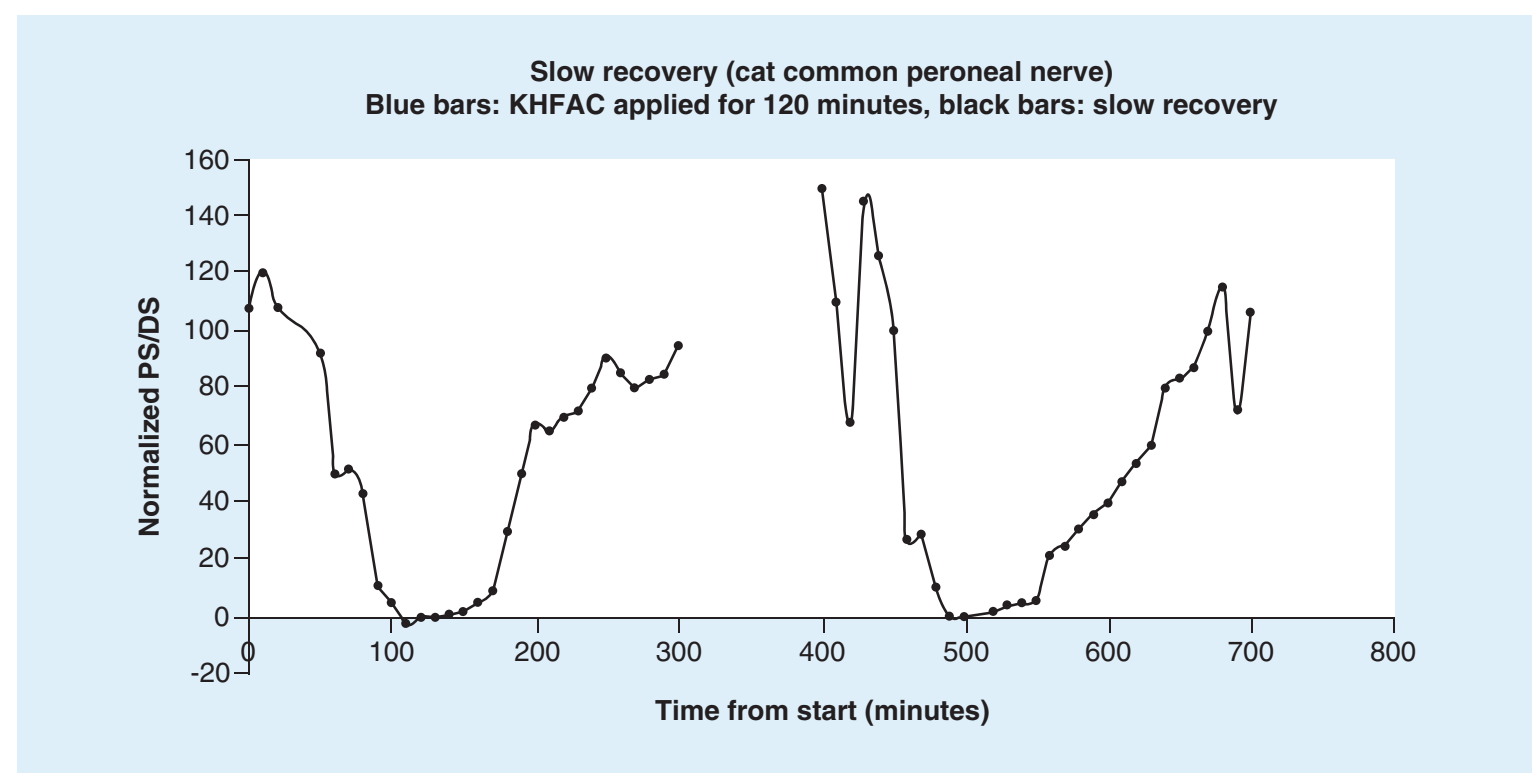

Figure 4. An example of slow recovery from carry-over block effect after application of kilohertz frequency alternating current for $\mathbf{2} \mathbf{h}$.

The PS/DS ratio is the ratio of twitch forces produced by test stimulation proximal and distal to the site of conduction block and is an index of residual nerve block and recovery.

COBE: 'Carry-over' block effect; DS: Distal stimulation; KHFAC: Kilohertz frequency alternating current; PS: Proximal stimulation. 
- It is feasible to use KHFAC to block different sizes of axons, from motor [3] and sensory fibers [20], to the smallest unmyelinated axons [23]. Tanner [7] described fiber size selectivity using a $20 \mathrm{kHz}$ KHFAC waveform. This result has also been confirmed for sensory nerves [20]. Patel and Butera [23,24] have shown that while motor fibers have lower thresholds for blocking than C-fibers at frequencies below $30 \mathrm{kHz}$, this relationship appears to be reversed at higher frequencies;

- The two important parameters of KHFAC nerve block are frequency and amplitude. Nerve conduction block requires a frequency of $1 \mathrm{kHz}$ or higher [13], and typically frequencies of $5 \mathrm{kHz}$ or higher have been used. There have been no reports of a 'maximum plateau' frequency to achieve KHFAC block, with the highest reported frequency being $50 \mathrm{kHz}$ [25].

KHFAC requires minimum amplitude, the 'block threshold', to produce complete block of the target axons [3]. Bhadra and Kilgore demonstrated that the block threshold is a linear function of KHFAC frequency over the range of 5-30 kHz. This relationship has also been established in other experiments, including cat pudendal [26], aplysia [24] and frog [23]. Recently, Butera [23] has suggested that the linear relationship may not be true for frequencies above $30 \mathrm{kHz}$ in C-fibers.

Both voltage-controlled KHFAC and current-controlled KHFAC waveforms have been used with no significant difference in the reported response [27]. However, DC offsets can be a significant issue, especially in current controlled waveforms, and care needs to be taken to utilize a circuit design that removes the DC offset [28].

- There are multiple theories presented regarding the mechanism of KHFAC block [10,29]. One theory is based on the accumulation of extracellular potassium [30]. However, KHFAC block has been shown to take effect too rapidly to support this theory [4]. Another theory has been that potassium currents flowing out overcome the inward sodium currents and produce block [12]. This theory has been based on some modeling studies [31]. However, there is no experimental confirmation of this theory and is unlikely to be the primary mechanism in mammalian nerve, since there are not many fast potassium channels in mammalian nodes [32].

A third theory focuses on the inactivation of sodium channel as the mechanism of KHFAC block $[3,29,33,34]$. Computation modeling studies indicated that KHFAC resulted in an increased inward sodium current, leading to a dynamic depolarization of many nodes under the electrode [33,35]. This led to the inactivation of about $90 \%$ of the sodium channels in the node directly under the electrode. There is some experimental evidence of this effect $[29,35]$.

- KHFAC has been utilized clinically in a few recently introduced medical applications. Enteromedics (MN, USA) has developed an obesity control application, the 'VBLOC' system, which uses a $5 \mathrm{kHz}$ KHFAC waveform applied to the vagal nerve [36]. A $10 \mathrm{kHz}$ KHFAC waveform is being applied for postamputation neuroma pain relief (Neuros Medical, Inc., OH, USA) with encouraging results [37]. Relief of back pain (Nevro Corp., CA, USA) has been obtained using a current-controlled $10 \mathrm{kHz}$ waveform delivered to the thoracic spinal cord [38].

KHFAC waveforms in these applications may produce the desired effect through direct nerve conduction block, or the effect may be through indirect means. It has not been verified that these systems are using KHFAC parameters that are sufficient to produce a direct conduction block.

\section{Electrical nerve block using DC \& CBPC}

DC block of neural activity was first demonstrated by Pflüger [39] and has been reviewed previously [40]. Until recently, however, the use of DC block was limited to physiological studies in acute animal preparations due to irreversibility of the block when utilized for more than a few minutes [41-43]. The reported amplitude necessary for complete block of a peripheral nerve varies widely, from a few microamperes [40,44] to many milliamperes [45]. These differences are due, at least in part, to the different electrode materials and designs used in these studies. Electrode materials used include saline-soaked felt, platinum of widths up to $6 \mathrm{~mm}$ and silver/silver chloride.

The mechanism of DC block is well understood and is based directly on the mechanisms of the ion channels to produce action potentials. In order to generate an action potential, the sodium channels must transition to an activated state through an increase in the membrane potential (depolarization). By hyperpolarizing the membrane it is possible to prevent the sodium channel from moving to an activated state. Once the membrane potential is increased high enough, the channel transitions to an inactivated state. By depolarizing the membrane, it is possible 
to hold the channel in the inactivated state. Thus DC of either polarity can produce a conduction block given the appropriate amplitude. Sassen and Zimmermann [46] demonstrated experimentally that hyperpolarization blocks at a much higher current than depolarization. Bhadra et al. [40] discovered, in both computational simulation and in vivo experiments, that a cathodic current will cause block at a lower threshold than an anodic current.

Electrical nerve block via DC can be achieved without initiating an action potential. Petruska et al. $[43,47]$ demonstrated that by ramping the amplitude of the DC over a period of approximately $100 \mathrm{~ms}$, it was possible to achieve block without inducing neural activation. This is in direct contrast to KHFAC which, as described earlier, always produces onset activity, even if the amplitude is ramped. The excitation at the cessation has likewise been eliminated using a ramp at the end of DC block $[43,47,48]$.

Historically, DC block has only been utilized in acute physiological testing because the electrochemical reactions at the electrode will eventually result in damage to both the nerve and electrode, and is thus unsuitable for long-term acute or chronic applications [42,49-51]. Charge can be delivered through an electrode through both capacitive and Faradaic reactions. Capacitive charge delivery occurs at low amplitudes and is reversible. Faradaic reactions typically occur at higher amplitudes and can be reversible, but if the reactants diffuse away from the electrode interface, these reactions become irreversible. Electrode corrosion and electrolysis of water can also occur. Irreversible reactions are the probable cause of nerve and electrode damage during DC delivery [52-54]. Because of these issues, safe electrical stimulation is achieved by limiting the charge delivery below established safety levels and delivering stimulation with a charge-balancing phase so that the waveform has a zero net charge. Safe levels of stimulation are well established $[55,56]$. For typical electrodes and amplitudes used in clinical applications, this criterion limits the duration of the active pulse to typically less than $1 \mathrm{~ms}$. However, applications utilizing electrical nerve block require the block to be continuously effective for seconds, minutes or even hours. Thus, if typical electrode designs and materials are used, DC block is impractical for any clinical applications.

Within the past few years, two approaches have been presented in the literature that describes the potential to utilize DC for nerve block. The first method has been termed the separated interface nerve electrode (SINE) and involves separating the electrode-electrolyte reactions from the nerve itself. The second method utilizes high charge capacity materials and maintains a charge balance over a period of many seconds to a minute or more. This latter method is referred to as CBPC. Both of these approaches have been demonstrated to significantly extend the duration that DC block can be reversibly applied in the acute situation (from tens of seconds for DC alone to many hours with CBPC) but neither method has yet been used chronically. These two methods are reviewed in the following paragraphs.

\section{Separated interface nerve electrode}

One possible approach for safely delivering DC to nerves would be to isolate the site of electrochemical reactions (at the electrode) from the nerves themselves. Ackermann et al. [57] evaluated this approach using a SINE. The SINE consisted of a syringe and tube filled with saline. The tube was connected to a cuff electrode surrounding the target nerve and an electrode was placed in the syringe. Current was delivered through the saline to the nerve to produce block. The results showed that long duration (up to $880 \mathrm{~s}$ ) DC pulses could be delivered to the nerve at amplitudes sufficient to block conduction and still maintain complete reversibility of block once the current was turned off [57]. More recently the SINE electrode has been enhanced to use carbon black in the electrolyte to extend the run time of the device. A 50 min block was achieved with complete nerve force recovery, as shown in Figure 5.

A modification of the SINE electrode has been developed by Fridman and Della Santina [58,59], called the safe DC stimulator (SDCS). The safe DC stimulator uses physical switching valves that are in phase with an alternating current square wave. The electrode delivers a constant DC ionic flow to the nerve while delivering a charge-balanced waveform to the electrode itself. It has been demonstrated that this approach can both inhibit and excite neural activity in the inner ear in chinchillas.

\section{CBPC system}

The CBPC approach takes advantage of the fact that electrode safety is primarily a factor of charge density [56]. The basic concept is that if it is possible to create an extremely large effective electrode surface area, it should be possible to deliver charge for many seconds and still avoid irreversible reactions. This approach also uses a second phase that balances the net charge. New electrode chemistries that increase the effective surface area of an electrode while maintaining the geometric area of the electrode have been evaluated for this purpose. Platinum black, for example, which is produced by electroplating platinum on platinum, has an effective surface area more than two 


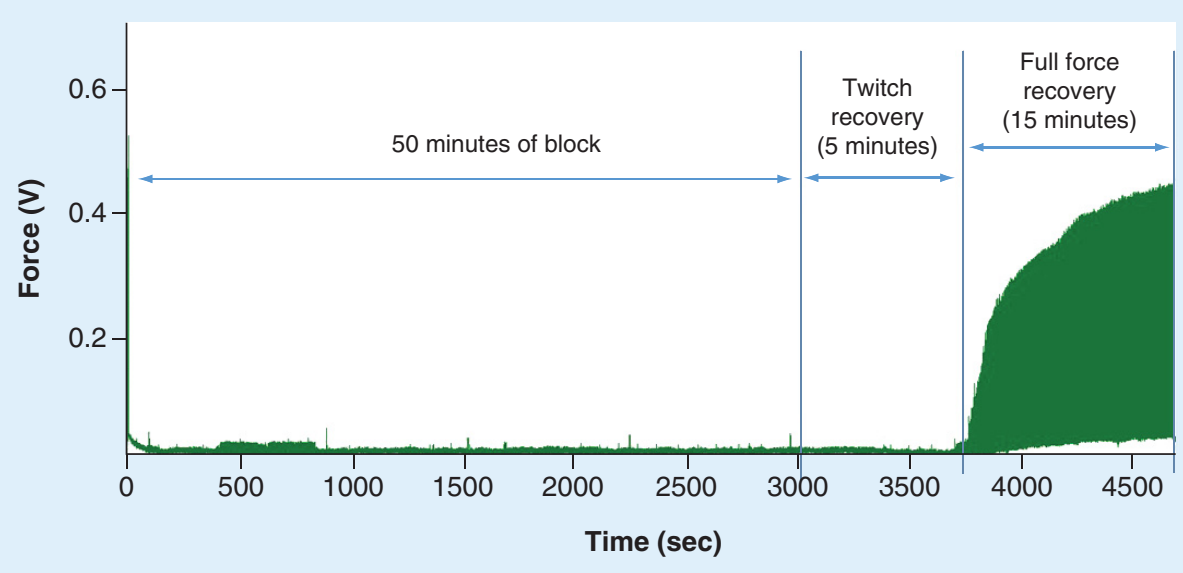

Figure 5. Enhanced carbon-based separated interface nerve electrode can provide block safely for $50 \mathrm{~min}$. The SINE is turned off after $50 \mathrm{~min}$ and conduction recovery begins $5 \mathrm{~min}$ after cessation of block. Full recovery of nerve conduction occurs 20 min after cessation of block.

SINE: Separated interface nerve electrode.

orders of magnitude larger than bare platinum [60]. Other materials such as Iridium oxide ( $\mathrm{IrOx}$ ) and carbon ink have also been used [61].

In order to take advantage of the capacitance of the electrode material, a biphasic waveform is used to reverse the reversible reactions. The biphasic waveform typically has a zero net charge. The phases do not need to be at the same current level, as long as the total charge is equal. The CBPC waveform using high charge capacity materials and biphasic waveforms can have a blocking phase as long as tens of seconds long [62].

Several different high capacitance electrode chemistries have been tested on the rat sciatic nerve to demonstrate both blocking ability and nerve integrity [60,61]. Proximal and distal stimulation (PS and DS) electrodes were placed on the sciatic nerve with a monopolar blocking electrode in between. The force generated by stimulating each of these electrodes was compared to determine block effectiveness and nerve integrity. If the force from the proximal electrode was reduced when the block was activated, then the block was effective. Block was achieved in all experiments. If the force generated from both the proximal and distal electrodes was the same, then nerve integrity was maintained. The ratio of PS to DS is used as metric for nerve damage. Figure 6 shows the effect of cumulative charge for bare platinum, platinum black and iridium oxide. The ratio of PS to DS was maintained for the high surface area material, but was reduced for the bare platinum. Carbon electrodes have been tested in several different applications. On the rat sciatic nerve, a four-contact electrode was tested to provide a long-term DC block. Block was applied for 22 min while maintaining nerve integrity, as shown in Figure 7 [63]. This same electrode configuration was tested on the paravertebral chain to blunt the sympathetic reflex to prevent cardiac arrhythmia induction [64].

The ability of DC block to provide complete block without activation of the onset response makes it an attractive option for many applications. The use of high capacitance electrodes has opened up the types of applications that can be developed to address a wide variety of needs.

\section{Electrical nerve block using combined waveforms}

As mentioned in the KHFAC section, mammalian experiments show that the KHFAC waveform produces a transient but intense activity in the nerve when it is first turned on, referred to as the 'onset response' [3]. One of the primary goals of research in electrical nerve block has been to understand the characteristics of the onset response, and to identify methods for reducing and eliminating this response while still maintaining the desirable characteristics of KHFAC block. The onset response is produced primarily by the response of the nerve to the first few cycles of the KHFAC wave [4]. In addition, lower amplitude KHFAC waves can produce repetitive firing in the nerve, and it is likely that regions of the nerve remote from the blocking site experience this condition. 


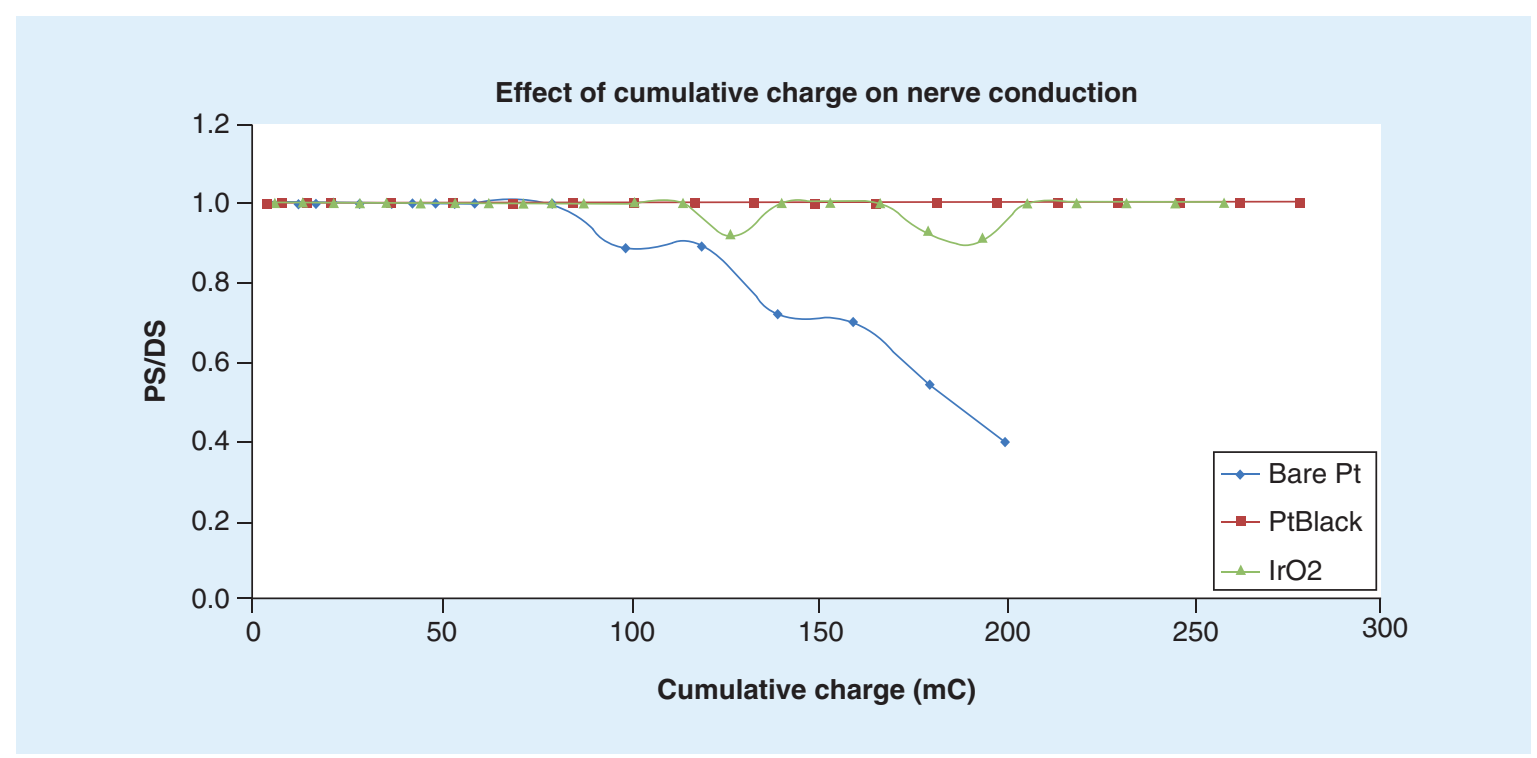

Figure 6. Change in proximal stimulation/distal stimulation ratio for different electrode chemistries. The nerve response degrades with bare platinum after approximately $70 \mathrm{mC}$ of charge delivery, whereas the nerve response is maintained at $100 \%$ for PtBlack and $\mathrm{IRO}_{2}$.

IRO2: Iridium oxide; PtBlack: Platinum black.

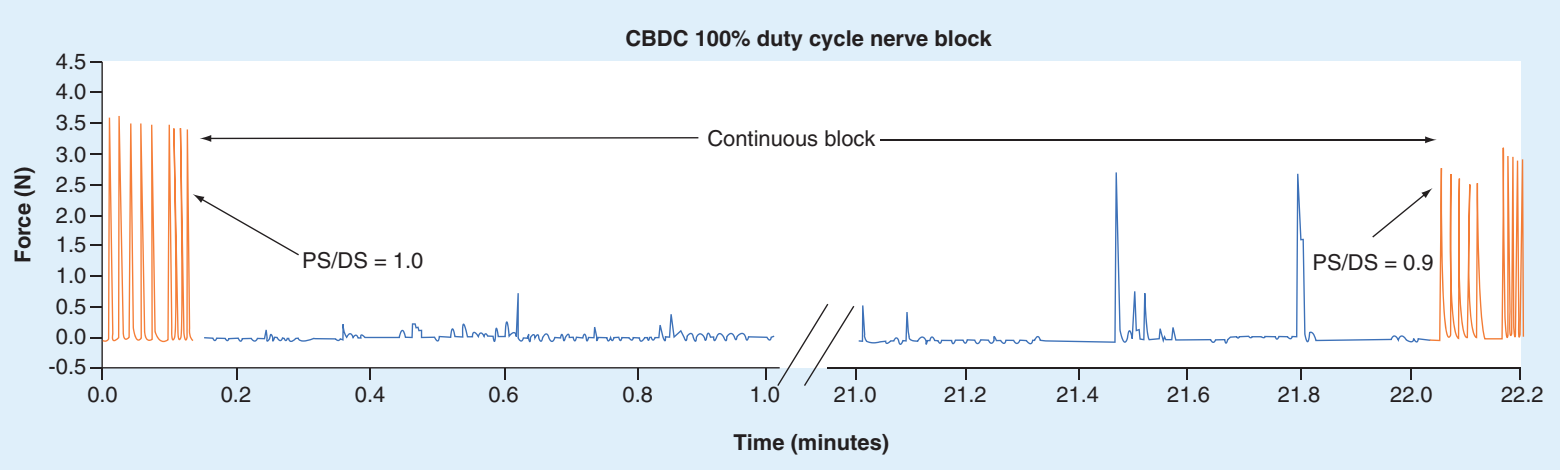

Figure 7. A four-contact electrode was used to maintain charge-balanced polarizing current block continuously by cycling through each contact. Block was applied continuously for $22 \mathrm{~min}$. Stimulation was applied at $1 \mathrm{~Hz}$ throughout the 22 -min period. Occasionally, action potentials can pass through the blocked region, typically during the transition of block from one contact to another (see occasional twitches during block period, as shown in blue). At the end of the block period, there is a slight carry-over of the block effect, as indicated by the decrease in the PS/DS ratio from 1.0 prior to block to 0.9 after block.

CBPC: Charge-balanced polarizing current; DS: Distal stimulation; PS: Proximal stimulation.

Ackermann et al. [21,22] demonstrated that the onset response can be reduced so that it lasts less than 1 or $2 \mathrm{~s}$ through optimization of electrode geometries and through the use of large KHFAC waveform amplitudes.

A common assumption has been that the onset activity could be eliminated by slowly ramping the amplitude of the KHFAC up to the threshold for block. However, it has been demonstrated that an amplitude ramp is unsuccessful because it is precisely the lower amplitudes where the onset activity is highest [65].

DC nerve block can also produce an onset response, but this can be eliminated by using an amplitude ramp with an optimal slope. Given the contrasting features, the concept of combining KHFAC and DC nerve blocks was proposed, with the goal of eliminating the onset response [66]. Through computational simulation, it was demonstrated that there is a continuum of electrical nerve block, spanning hyperpolarizing DC block, charge balanced KHFAC block and depolarizing DC block, as outlined in Figure 8 [67]. Most electrical block occurs 


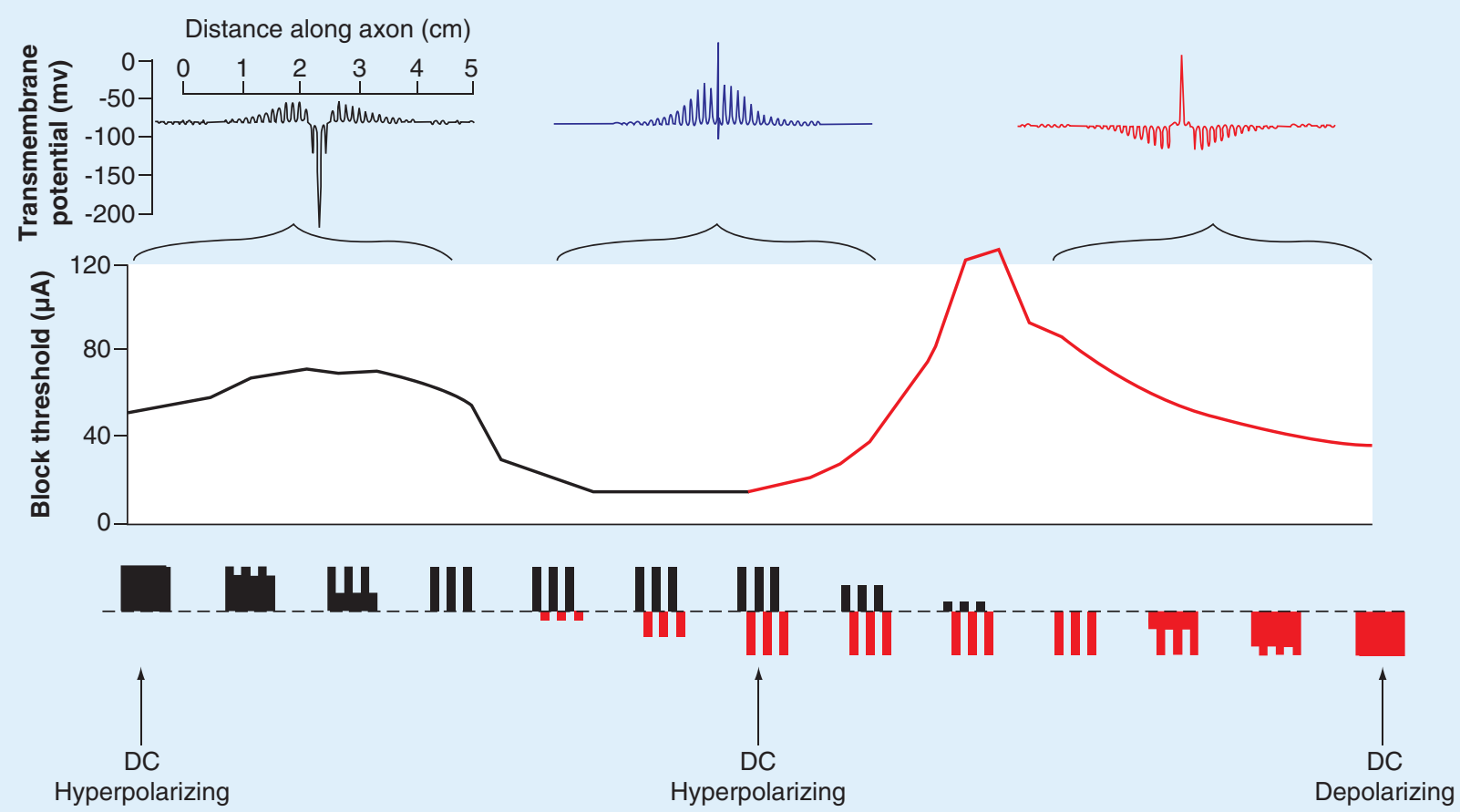

Figure 8. Unifying theory of electrical nerve conduction block showing results from computer simulations. The waveform varies from (left to right) anodal DC, anodal monophasic pulses, charged-balanced alternating current, cathodal monophasic pulses and cathodal DC, as shown below the $\mathrm{x}$-axis (black = anodic; red = cathodic). There are three regions of block: hyperpolarizing, alternating current and depolarizing. The graph indicates the block threshold for each waveform along the continuum. Three insets along the top of the graph illustrate transmembrane voltage with the axonal length along the x-axis. Depolarization is upwards. On the left is 'virtual depolarization block', in the center is 'KHFAC block from dynamic depolarization' and on the right is 'true depolarization block'.

DC: Direct current; KHFAC: Kilohertz frequency alternating current.

through depolarization mechanisms of the axonal membrane and the onset mitigation/elimination strategies rely on DC depolarization nerve block.

One approach to combining CBPC and KHFAC is to deliver each waveform through separate electrodes [66,68]. With this approach, CBPC block is first established on two flanking electrodes (without producing an onset). Once block is established, KHFAC is delivered through an electrode positioned between the two flanking CBPC electrodes. In this manner the onset response is blocked so that it does not propagate beyond the electrodes. Once KHFAC block is in effect with termination of the onset response, it is then possible to turn the CBPC electrodes off. An example of the results using this approach is shown in Figure 9.

A second approach has the advantage of combining the CBPC and KHFAC through the same electrode [69]. This waveform was developed through computer simulations of waveforms that combine a transient period of charge imbalance with the KHFAC waveform. The waveform is referred to as the combined no onset waveform [69]. The waveform begins with a DC portion of the waveform that ensures that there is no onset response. KHFAC is then initiated at low amplitude on top of the DC portion. Both the DC and KHFAC are then modulated so that no onset response is produced, culminating in a waveform that delivers KHFAC only, which can then be used to maintain block for up to as much as 40 min without carry-over effects. Figure 10 shows an example of the results of the combined no onset waveform, producing an effective block without an onset in the rat sciatic nerve.

\section{Waveforms often associated with electrical nerve block}

The application of electrical current to neural structures can have a variety of physiological effects based on the neural structures affected by the electrical current, the stimulation parameters, electrode geometry, electrode materials and many other factors (e.g., Figure 3, showing the effect of KHFAC as a function of amplitude and duration). This has led to significant confusion in the literature, particularly with respect to electrical nerve block. Similar terms have been used to describe very different effects and, in some cases, very different electrical parameters produce 

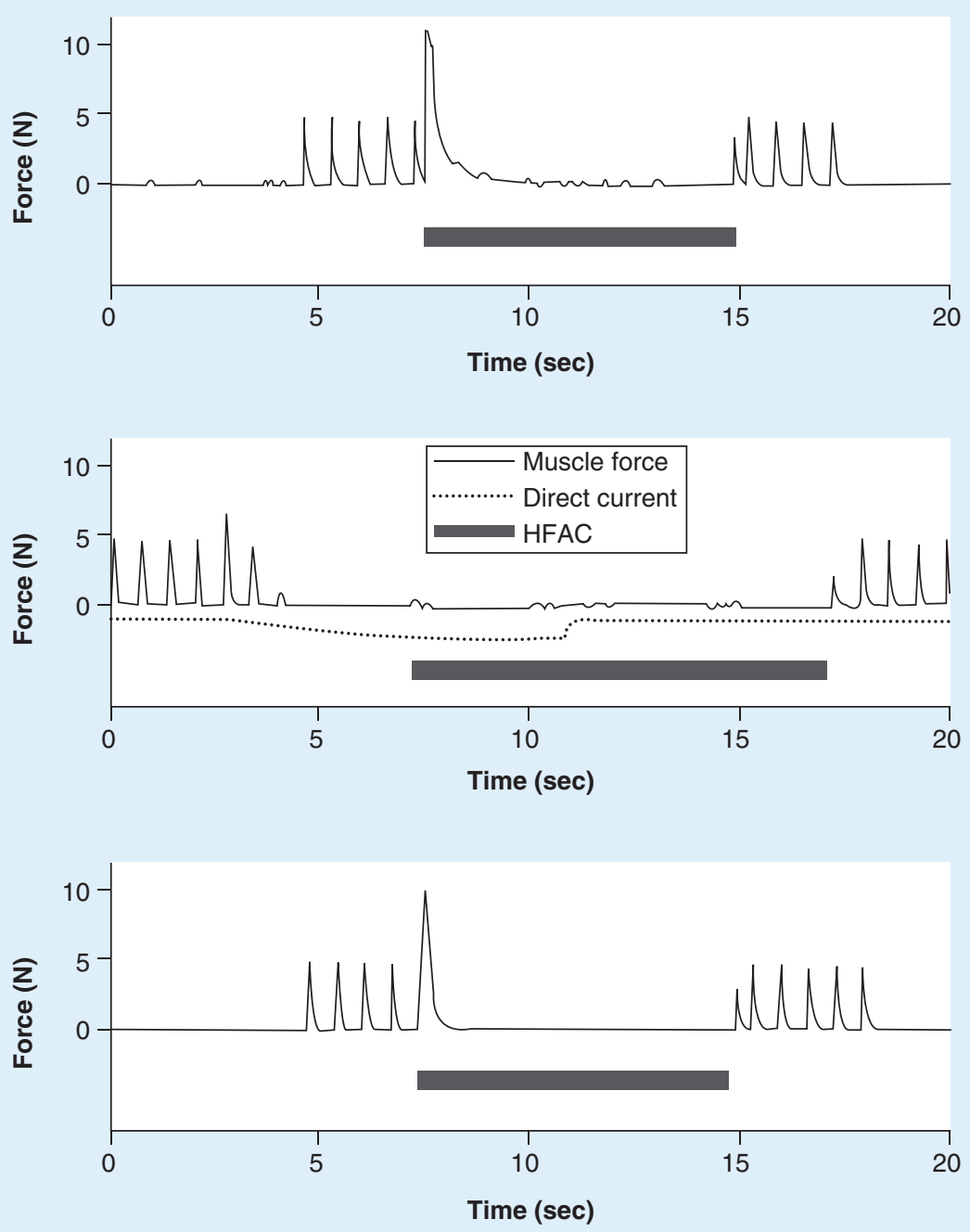

Figure 9. Example of combined direct current and kilohertz frequency alternating current block with separate electrodes. KHFAC is the first to be tested alone to evaluate KHFAC onset. Next the DC is added to block the onset. KHFAC is then applied again alone to verify that the KHFAC onset had remained constant. DC: Direct current; KHFAC: Kilohertz frequency alternating current.

very similar effects. For example, as we have reviewed in this manuscript, KHFAC and CBPC can both produce a block of action potential conduction in nerves, yet the parameters used are many orders of magnitude different from one another. Without a direct measure of action potential block, it can be very difficult to discern whether an actual block has occurred even if electrical parameters are used that might produce an electrical conduction block in the right conditions. In this section, we will briefly review a few of the key misunderstandings and seek to clarify the interpretation of the published results.

One unfortunate assumption that is often made is that electrical nerve block is synonymous with 'high frequency' stimulation, where high frequency is any frequency that is higher, on a relative basis, than the frequencies typically used. However, as we have reviewed above, although KHFAC can produce electrical nerve conduction block (see specific definition in the Introduction), it only does so under specific conditions related to electrical parameters, geometry between electrical delivery and nerve and the targeted neural structures. Devices that deliver a kilohertz frequency to a nerve may actually activate rather than block. Without a direct test of nerve conduction block, it is difficult, if not impossible to discern the effect. An example of this is the use of $10 \mathrm{kHz}$ waveform for spinal cord stimulation [38]. Initially it was commonly thought that this waveform produced a conduction block of fibers [25,70], but subsequent studies have shown that this is unlikely to be the mechanism of action for this particular device $[71,72]$. 

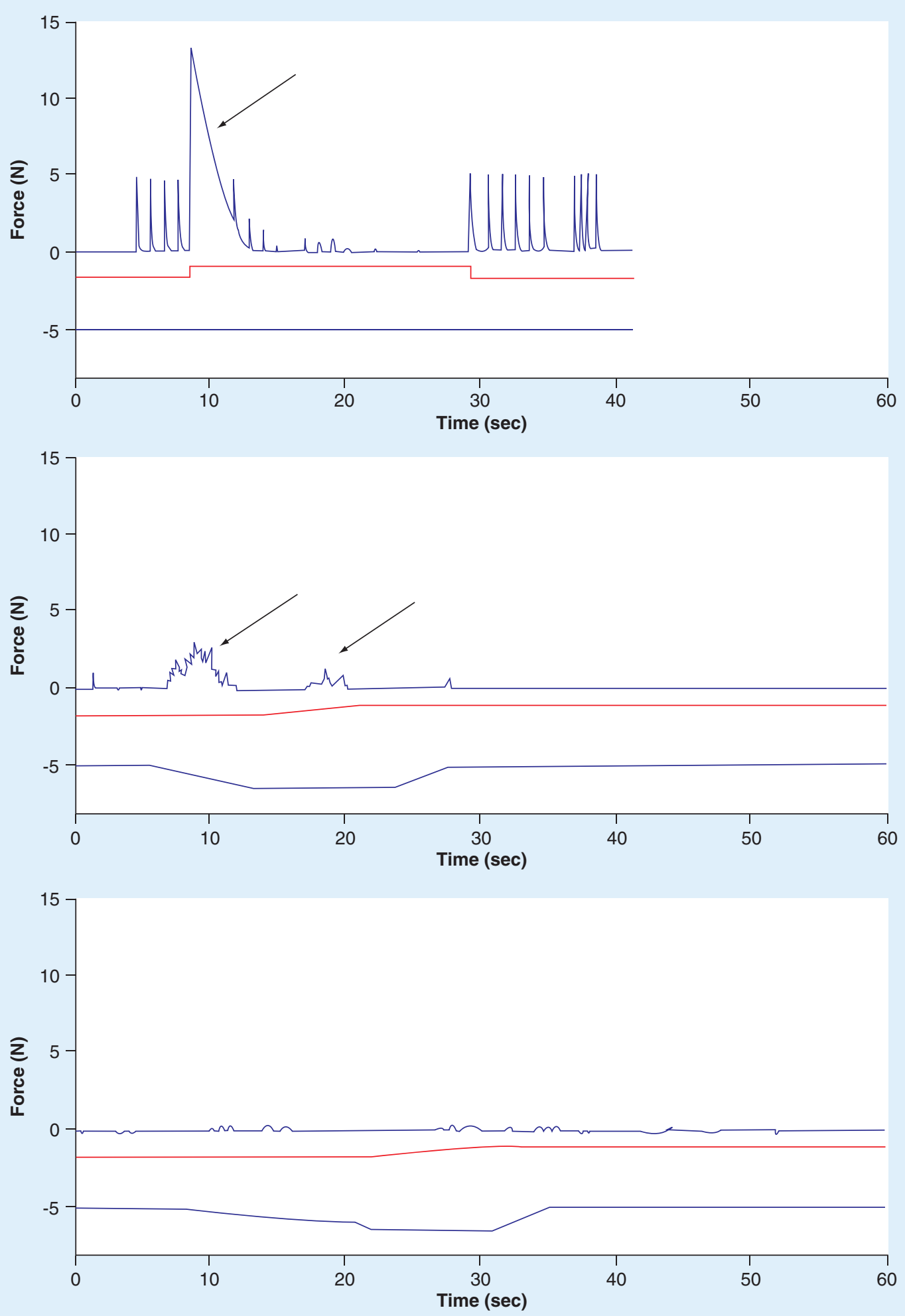

Figure 10. In vivo results of the combined no onset waveform that initiates block with a pure direct current depolarization ramp and then gradually mixes in the kilohertz frequency alternating current before terminating the direct current with another ramp. The ramps prevent any onsets from the DC. A $7 \mathrm{~mA} 20 \mathrm{KHz}$ sine wave was applied to the nerve which generated an onset (arrow) with a peak force of $13.5 \mathrm{~N}$ (top). The DC block threshold was determined to be -1.4 mA. A DC signal at this level was added to the KHFAC signal (bottom blue trace in each figure). This mitigation strategy reduced the onset peak force to $3.0 \mathrm{~N}$ (middle, arrows). The KHFAC signal was set to $7 \mathrm{~mA}$ and the ramp to DC and the ramp to recharge were increased. This further decreased the peak onset to $0.4 \mathrm{~N}$ (bottom). The CNOW mitigation successfully reduced the onset to $3 \%$ of the peak force compared with the KHFAC alone. CNOW: Combined no onset waveform; DC: Direct current; KHFAC: Kilohertz frequency alternating current. 
The generation of KHFAC waveforms can be difficult to achieve without introducing a slight charge imbalance at the electrode-nerve interface [28]. This could alter the effect of the KHFAC on the nerve so that it is effectively closer to the effect of a DC waveform, with possible implications on long-term effects at both the nerve and electrode. An extreme example of this was the use of a monophasic waveform by Solomonow et al. [73-76]. This group evaluated a broad frequency range, identifying $600 \mathrm{~Hz}$ with a pulse width lasting 50-100 $\mu$ s as the optimum block frequency. From a physiological perspective, a monophasic waveform is effectively an 'interrupted DC' waveform. The mechanism of action of this waveform is likely to be related to the DC effect. Unfortunately, since the frequency range used $(500-10 \mathrm{kHz})$ was considered 'high frequency', it is often confused with electrical nerve block using KHFAC. It is more appropriate, however, to classify it as a DC-type of electrical nerve block. In addition, based on the experimental design used in these studies, it is also likely that the 'block' achieved was due to transient depletion of the neurotransmitter at the neuromuscular junction [3].

A more recently introduced waveform used to provide pain relief is the 'burst' waveform [77]. This waveform consists of brief packets or bursts of 'high' frequency pulses (typically $\sim 500 \mathrm{~Hz}$ ) delivered at a much lower base frequency (typically $\sim 50 \mathrm{~Hz}$ ). Because of the high frequency label, this waveform is also often categorized with other KHFAC waveforms. However, the $500 \mathrm{~Hz}$ pulses are delivered in a monophasic fashion with a recharge phase delivered after the burst is complete. Given the monophasic nature, the $500 \mathrm{~Hz}$ pulses act closer to a single pulse. Also, $500 \mathrm{~Hz}$ is well below the frequency range necessary to achieve nerve conduction block [13]. The burst waveform may have a different mechanism of action than the other waveforms we have reviewed here, but there is no evidence that it produces a nerve conduction block.

Most of the waveforms described in this section were not necessarily developed to achieve the specifically defined 'electrical nerve block' as we have defined it. Nevertheless, these waveforms often get categorized together when they are, in fact, very distinct modalities. Progress in this field of research can be significantly enhanced by specifically defining the targeted effects (electrical nerve block, neuromuscular depletion block and neuromodulation, etc.) and careful demonstration that the targeted effects do, in fact, occur (ideally through demonstration of the effect at the local neuronal level rather than using a broadly systemic outcome, such as pain relief or weight loss).

\section{Conclusion \& future perspective}

Electrical nerve block is a field still in its infancy when compared with electrical stimulation. Issues such as a typical range of parameters, established safety limits and desirable materials and geometries are well established for electrical stimulation but are, as yet, undetermined for electrical nerve block. In the near future, chronic safety studies will need to be conducted in order to establish the safety of each approach to nerve block. This will significantly expand the opportunities to test nerve block in human subjects and for targeted diseases. Given the need for CBPC nerve block to use high charge capacity materials, it is likely that new electrode materials will be developed that will prove more durable and with good biocompatibility profiles when compared with the materials that have been used to date (Pt-black, carbon ink and $\mathrm{IrOx}$ ). Without the completion of these basic components of an electrical nerve block system, it will be difficult to proceed to widespread clinical application.

A key area of future research will involve assessing the differing effect of electrical nerve block parameters on the full range of neuron diameters, from large myelinated $\alpha$-fibers to small unmyelinated C-fibers. To date, there is no clear resolution to this issue. Computational simulations suggest that KHFAC block is more likely to block large fibers [3,71]. Experimental results suggest that this effect may reverse at high frequencies (i.e., $>50 \mathrm{kHz}$ ) [24]. However, these experiments are difficult to conduct and are likely affected by specific electrode design and the characteristics of the waveform generator. Further studies examining this effect are warranted. In addition, exploring methods of altering the nerve block waveforms or electrodes to enhance the blocking effect in small fibers is highly desirable. In the ideal case, a system that could preferentially block small and large fibers independently would provide significant flexibility for a wide array of clinical applications.

The mechanism of action of different types of electrode nerve block will continue to be explored in both computational and experimental studies. A better understanding of the mechanism of action should allow optimization of the waveform parameters. In addition, understanding the mechanism of action of related waveforms (such as burst, HF10, etc.) will help guide the field to be able to properly categorize the differing neuronal effects. This should help to clear up the misunderstandings that still persist.

We anticipate that the clinical applications of nerve block will initially target diseases for which the treatment of last resort involves denervating techniques (surgical or chemical), such as treatment of muscle spasticity in cervical dystonia, cerebral palsy and stroke. As experience is gained with this technique, application will certainly expand to 
the acute and chronic pain domains. In particular, the features of quick action and quick reversibility may be well suited to applications of acute pain, such as postoperative nerve pain. However, the most advanced and exciting applications of electrical nerve block are likely to be in the control of autonomic functions, where the ability to downregulate nerve activity is a key component for treating diseases such as heart failure, chronic obstructive pulmonary disease and hypertension. Such systems are likely to combine electrical (or other modalities) activation of nerves with electrical inactivation of nerves to gain nearly complete control over nervous system function.

In summary, electrical nerve block is an emerging technique that has many potential significant advantages over other methods of nerve block. Electrical nerve block is rapidly acting and rapidly reversing. It can be titrated in real time. It can be used to target specific nerves with no systemic effects and a minimum of side effects. This field is still in its infancy, but a significant expansion in the clinical application of this technique is expected in the next few years.

\section{Executive summary}

- The new field of electrical nerve block, in which electrical waveforms are utilized to block action potential propagation, opens up unique applications in bioelectronics.

- Electrical nerve block has two key features that distinguish it from other nonelectrical means of nerve block. First, electrical nerve block occurs instantly, often on an action-potential by action-potential basis. Second, electrical nerve block is fully and rapidly reversible, and typically the nerve returns to preblock conduction within seconds after the cessation of the waveform delivery.

- Two approaches to electrical nerve block have emerged: kilohertz frequency alternating current (KHFAC) and direct current (DC) or modified DC (charge-balanced polarizing current).

- KHFAC is typically applied to a peripheral nerve through a nerve cuff electrode and can produce a continuous block of nerve conduction for tens of minutes at a time. Methods of DC block require the correct electrode materials and waveforms in order to safely and repeatedly block nerve conduction.

- One disadvantage of KHFAC is that it produces an 'onset response', which is a period of intense neural activity that occurs when the KHFAC is first delivered to the nerve. DC block can be established without the onset response. As a result, methods of combining these two approaches to achieve the advantageous features of each have been proposed.

- There is some confusion in the literature with respect to the varying physiological effects of electrical waveforms. High frequency waveforms are often equated with electrical nerve block when, in fact, these waveforms do not produce the same neural effects and do not directly block action potential conduction. Without a direct measure of action potential block, it can be very difficult to discern whether an actual block has occurred even if electrical parameters are used that might produce an electrical conduction block in the right conditions.

- Electrical nerve block is an emerging technique that has many potential significant advantages over other methods of nerve block. This field is still in its infancy, but a significant expansion in the clinical application of this technique is expected in the next few years.

\section{Financial \& competing interests disclosure}

This work was supported by NIH NINDS R01-NS-074149, NIBIB R01-EB-002091 and the Case Coulter Translation \& Innovation Partnership. K Kilgore and N Bhadra have equity ownership in Neuros Medical, Inc. The authors have no other relevant affiliations or financial involvement with any organization or entity with a financial interest in or financial conflict with the subject matter or materials discussed in the manuscript apart from those disclosed.

No writing assistance was utilized in the production of this manuscript.

\section{References}

1 Hambrecht FT. Functional electrical-stimulation - an overview. Pace-Pacing and Clinical Electrophysiol. 12(5), 840-843 (1989).

2 Linderoth B, Foreman RD. Physiology of spinal cord stimulation: review and update. Neuromodulation 2(3), 150-164 (1999).

3 Bhadra N, Kilgore KL. High-frequency electrical conduction block of mammalian peripheral motor nerve. Muscle Nerve 32(6), 782-790 (2005).

4 Foldes EL, Ackermann D, Bhadra N, Kilgore KL. Counted cycles method to quantify the onset response in high-frequency peripheral nerve block. Conf. Proc. IEEE Eng. Med. Biol. Soc. 2009, 614-617 (2009).

5 Bugnard L, Hill AV. High-frequency stimulation of nerve and the refractory period. J. Physiol. 83(4), 383-393 (1935). 
6 Cattell M, Gerard RW. The 'inhibitory' effect of high-frequency stimulation and the excitation state of nerve. J. Physiol. 83(4), 407-415 (1935).

7 Tanner JA. Reversible blocking of nerve conduction by alternating current excitation. Nature 195, 712-713 (1962).

8 Woo MY, Campbell B. Asynchronous firing and block of peripheral nerve conduction by $20 \mathrm{Kc}$ alternating current. Bull. Los Angel Neuro. Soc. 29, 87-94 (1964).

9 Bowman BR, Mcneal DR. Response of single alpha motoneurons to high-frequency pulse trains. Firing behavior and conduction block phenomenon. Appl. Neurophysiol. 49(3), 121-138 (1986).

10 Kilgore KL, Bhadra N. Reversible nerve conduction block using kilohertz frequency alternating current. Neuromodulation doi:10.1111/ner.12100 (2013) (Epub ahead of print).

11 Bhadra N, Kilgore KL. High-frequency nerve conduction block. Conf. Proc. IEEE Eng. Med. Biol. Soc. 7, 4729-4732 (2004).

12 Tai C, De Groat WC, Roppolo JR. Simulation analysis of conduction block in unmyelinated axons induced by high-frequency biphasic electrical currents. IEEE Trans. Biomed. Eng. 52(7), 1323-1332 (2005).

13 Williamson RP, Andrews BJ. Localized electrical nerve blocking. IEEE Trans. Biomed. Eng. 52(3), 362-370 (2005).

14 Clark JD. Spinal cord stimulation does frequency matter? Anesthesiol. 119(2), 243-244 (2013).

15 Buckley U, Chui RW, Rajendran PS, Vrabec T, Shivkumar K, Ardell JL. Bioelectronic neuromodulation of the paravertebral cardiac efferent sympathetic outflow and its effect on ventricular electrical indices. Heart Rhythm 14(7), 1063-1070 (2017).

16 Kilgore KL, Bhadra N. High frequency mammalian nerve conduction block: simulations and experiments. Conf. Proc. IEEE Eng. Med. Biol. Soc. 1, 4971-4974 (2006).

17 Waataja JJ, Tweden KS, Honda CN. Effects of high-frequency alternating current on axonal conduction through the vagus nerve. J. Neural Eng. 8(5), 056013 (2011).

18 Bhadra N, Vrabec T, Bhadra N, Kilgore KL. Response of peripheral nerve to high frequency alternating current (HFAC) nerve block applied for long durations. Presented at: Neural Interface Conference. Salt Lake City, UT, USA, 18-20 June (2012).

19 Bhadra N, Foldes EL, Ackermann D, Kilgore KL. Reduction of the onset response in high frequency nerve block with amplitude ramps from non-zero amplitudes.. IEEE Eng. Med. Biol. Soc. 650-653 (2009).

20 Lahowetz EL, Bhadra N, Kilgore KL. High frequency conduction block of sensory nerves. Presented at: 12th Annual Conference of the International Functional Electical Stimulation Society. Philadelphia, PA, USA (November 2007).

21 Ackermann DM Jr, Bhadra N, Foldes EL, Wang XF, Kilgore KL. Effect of nerve cuff electrode geometry on onset response firing in high-frequency nerve conduction block. IEEE Trans. Neural Syst. Rehabil. Eng. 18(6), 658-665 (2010).

22 Ackermann D, Foldes EL, Bhadra N, Kilgore KL. Electrode design for high frequency block: effect of bipolar separation on block thresholds and the onset response. Conf Proc IEEE Eng Med Biol Soc 2009, 654-657 (2009).

23 Joseph L, Butera RJ. High-frequency stimulation selectively blocks different types of fibers in frog sciatic nerve. IEEE Trans. Neural Syst. Rehabil. Eng. 19(5), 550-557 (2011).

24 Joseph L, Butera RJ. Unmyelinated Aplysia nerves exhibit a nonmonotonic blocking response to high-frequency stimulation. IEEE Trans. Neural Syst. Rehabil. Eng. 17(6), 537-544 (2009).

25 Cuellar JM, Alataris K, Walker A, Yeomans DC, Antognini JF. Effect of high-frequency alternating current on spinal afferent nociceptive transmission. Neuromodulation doi:10.1111/ner.12015 (2012) (Epub ahead of print).

26 Bhadra N, Kilgore K, Gustafson KJ. High frequency electrical conduction block of the pudendal nerve. J. Neural. Eng. 3(2), 180-187 (2006).

27 Kilgore K, Bhadra N. Nerve conduction block utilising high-frequency alternating current. Med. Biological Eng. Comput.42(3), (2004).

28 Franke M, Bhadra N, Bhadra N, Kilgore K. Direct current contamination of kilohertz frequency alternating current waveforms. J. Neurosci. Methods 232, 74-83 (2014).

29 Ackermann DM, Bhadra N, Gerges M, Thomas PJ. Dynamics and sensitivity analysis of high-frequency conduction block. J. Neural. Eng. 8(6), 065007 (2011).

30 Tai C, Guo D, Wang J, Roppolo JR, De Groat WC. Mechanism of conduction block in amphibian myelinated axon induced by biphasic electrical current at ultra-high frequency. J. Comput. Neurosci. 31(3), 615-623 (2011).

31 Tai C, De Groat WC, Roppolo JR. Simulation of nerve block by high-frequency sinusoidal electrical current based on the Hodgkin-Huxley model. IEEE Trans. Neural Syst. Rehabil. Eng. 13(3), 415-422 (2005).

32 Schwarz JR, Reid G, Bostock H. Action potentials and membrane currents in the human node of Ranvier. Pflugers Arch. 430(2), 283-292 (1995).

33 Bhadra N, Lahowetz EA, Foldes ST, Kilgore KL. Simulation of high-frequency sinusoidal electrical block of mammalian myelinated axons. J. Comput. Neurosci. 22(3), 313-326 (2007).

34 Elbasiouny SM, Mushahwar VK. Modulation of motoneuronal firing behavior after spinal cord injury using intraspinal microstimulation current pulses: a modeling study. J. Appl. Physiol. 103(1), 276-286 (2007). 
35 Bromm B. Spike frequency of the nodal membrane generated by high-frequency alternating current. Pfiügers Archiv. Eur. J. Physiol. 353(1), 1-19 (1975).

36 Camilleri M, Toouli J, Herrera MF et al. Selection of electrical algorithms to treat obesity with intermittent vagal block using an implantable medical device. Surg. Obes. Relat. Dis. 5(2), 224-229; discussion 229-230 (2009).

37 Soin A. Long-term human testing of high-frequency nerve block for amputation pain. Presented at: 16th Annual Meeting North American Neuromodulation Society. Las Vegas, Nevada, USA (2012).

38 Van Buyten JP, Al-Kaisy A, Smet I, Palmisani S, Smith T. High-frequency spinal cord stimulation for the treatment of chronic back pain patients: results of a prospective multicenter European clinical study. Neuromodulation 16(1), 59-66 (2013).

39 Pflüger EFW. Untersuchungen uber die Physiologie des Electrotonus. Hirschwald, Berlin, Germany (1858).

40 Bhadra N, Kilgore KL. Direct current electrical conduction block of peripheral nerve. IEEE Trans. Neural Syst. Rehabil. Eng. 12(3), 313-324 (2004).

41 Kuffler SW, Gerard RW. The small-nerve motor system to skeletal muscle. J. Neurophysiol. 10(6), 383-394 (1947).

42 Whitman JG, Kidd C. The use of direct current to cause selective block of large fibres in peripheral nerves. Br. J. Anaesth. 47(11), 1123-1133 (1975).

43 Petruska JC, Hubscher CH, Johnson RD. Anodally focused polarization of peripheral nerve allows discrimination of myelinated and unmyelinated fiber input to brainstem nuclei. Exp. Brain Res. 121(4), 379-390 (1998).

44 Sassen M, Zimmerma M. Differential blocking of myelinated nerve fibers by transient depolarization. Pflugers Archiv-Eur. J. Physiol. 341(3), 179-195 (1973).

45 Accornero N, Bini G, Lenzi GL, Manfredi M. Selective Activation of peripheral nerve fibre groups of different diameter by triangular shaped stimulus pulses. J. Physiol. 273(3), 539-560 (1977).

46 Sassen M, Zimmermann M. Differential blocking of myelinated nerve fibres by transient depolarization. Pflugers Archiv. Eur. J. Physiol. 341(3), 179-195 (1973).

47 Zimmermann M. Selective activation of C-fibers. Pflugers Arch. Gesamte Physiol. Menschen. Tiere 301(4), 329-333 (1968).

48 Sweeney JD, Mortimer JT. An asymmetric two electrode cuff for generation of unidirectionally propagated action potentials. IEEE Transactions on Bio-medical Engineering 33(6), 541-549 (1986).

49 Manfredi M. Differential block of conduction of larger fibers in peripheral nerve by direct current. Arch. Ital. Biol. 108(1), 52-71 (1970).

50 Merrill DR, Bikson M, Jefferys JG. Electrical stimulation of excitable tissue: design of efficacious and safe protocols. J. Neurosci. Methods 141(2), 171-198 (2005).

51 Guz A, Trenchard DW. The role of nonmyelinated vagal afferent fibres from the lungs in the genesis of tachypnoea in the rabbit. J. Physiol. 213(2), 345-371 (1971).

52 Brummer SB, Turner MJ. Electrical stimulation with Pt electrodes: II-estimation of maximum surface redox (theoretical non-gassing) limits. IEEE Trans. Biomed. Eng. 24(5), 440-443 (1977).

53 Mccreery DB, Agnew WF, Yuen TG, Bullara LA. Comparison of neural damage induced by electrical stimulation with faradaic and capacitor electrodes. Ann. Biomed. Eng. 16(5), 463-481 (1988).

54 Ravid E, Prochazka A. Controlled nerve ablation with direct current: parameters and mechanisms. IEEE Trans. Neural Syst. Rehabil. Eng. doi:10.1109/TNSRE.2014.2307756 (2014) (Epub ahead of print).

55 Mccreery DB, Agnew WF, Yuen TGH, Bullara L. Charge-density and charge per phase as cofactors in neural injury induced by electrical-stimulation. IEEE Trans. Biomed. Eng. 37(10), 996-1001 (1990).

56 Shannon RV. A model of safe levels for electrical stimulation. IEEE Trans. Biomed. Eng. 39(4), 424-426 (1992).

57 Ackermann DM Jr, Bhadra N, Foldes EL, Kilgore KL. Separated interface nerve electrode prevents direct current induced nerve damage. J. Neurosci. Methods 201(1), 173-176 (2011).

58 Fridman GY, Della Santina CC. Safe direct current stimulation to expand capabilities of neural prostheses. IEEE Trans. Neural Syst. Rehabil. Eng. 21(2), 319-328 (2013).

59 Fridman GY, Della Santina CC. Safe direct current stimulator 2: concept and design. Conf. Proc. IEEE Eng. Med. Biol. Soc. 2013, 3126-3129 (2013).

60 Vrabec T, Bhadra N, Wainright J, Bhadra N, Franke M, Kilgore K. Characterization of high capacitance electrodes for the application of direct current electrical nerve block. Medical \& Biological Engineering \& Computing doi:10.1007/s11517-015-1385-5 1-13 (2015) (Epub ahead of print).

61 Vrabec TL. Direct current block of peripheral nerve: electrode and waveform development. PhD Dissertation, Dept. of Biomedical Engineering, Case Western Reserve University (2016).

62 Vrabec T, Bhadra N, Wainright J, Bhadra N, Franke M, Kilgore K. Characterization of high capacitance electrodes for the application of direct current electrical nerve block. Med. Biol. Eng. Comput. doi:10.1007/s11517-015-1385-5 (2015) (Epub ahead of print). 
63 Vrabec T, Bhadra N, Acker G, Bhadra N, Kilgore K. Continuous direct current nerve block using multi contact high capacitance electrodes. IEEE Transactions on Neural Systems and Rehabilitation Engineering PP(99), 1-1 (2016).

64 Chui RW, Buckley U, Rajendran PS, Vrabec T, Shivkumar K, Ardell JL. Bioelectronic block of paravertebral sympathetic nerves mitigates post-myocardial infarction ventricular arrhythmias. Heart Rhythm 14 (11)1665-1672 (2017).

65 Miles JD, Kilgore KL, Bhadra N, Lahowetz EA. Effects of ramped amplitude waveforms on the onset response of high-frequency mammalian nerve block. J. Neural. Eng. 4(4), 390-398 (2007)

66 Ackermann DM Jr, Bhadra N, Foldes EL, Kilgore KL. Conduction block of whole nerve without onset firing using combined high frequency and direct current. Med. Biol. Eng. Comput. 49(2), 241-251 (2011).

67 Kilgore KL, Bhadra N. Reversible nerve conduction block using kilohertz frequency alternating current. Neuromodulation 17(3), 242-255 (2014)

68 Franke M, Vrabec T, Wainright J, Bhadra N, Bhadra N, Kilgore K. Combined KHFAC + DC nerve block without onset or reduced nerve conductivity after block. J. Neural. Eng. 11(5), 056012 (2014).

69 Vrabec TL, Bhadra N, Wainright JS, Bhadra N, Kilgore KL. A novel waveform for no-onset nerve block combining direct current and kilohertz frequency alternating current. 2013 6th International IEEE/EMBS Conference on Neural Engineering (Ner) 283-286 (2013).

70 Arle JE, Mei LZ, Carlson KW, Shils JL. High-frequency stimulation of dorsal column axons: potential underlying mechanism of paresthesia-free neuropathic pain relief. Neuromodulation 19(4), 385-397 (2016).

71 Lempka SF, Mcintyre CC, Kilgore KL, Machado AG. Computational analysis of kilohertz frequency spinal cord stimulation for chronic pain management. Anesthesiology 122(6), 1362-1376 (2015)

72 Crosby ND, Janik JJ, Grill WM. Modulation of activity and conduction in single dorsal column axons by kilohertz-frequency spinal cord stimulation. J. Neurophysiol. 117(1), 136-147 (2017).

73 Solomonow M. External control of the neuromuscular system. IEEE Trans. Biomed. Eng. 31(12), 752-763 (1984).

74 Solomonow M, Eldred E, Lyman J, Foster J. Fatigue considerations of muscle contractile force during high-frequency stimulation. Am. J. Phys. Med. 62(3), 117-122 (1983).

75 Zhou BH, Baratta R, Solomonow M. Manipulation of muscle force with various firing rate and recruitment control strategies. IEEE Trans. Biomed. Eng. 34(2), 128-139 (1987).

76 Baratta R, Ichie M, Hwang SK, Solomonow M. Orderly stimulation of skeletal-muscle motor units with tripolar nerve cuff electrode. IEEE Trans. Biomed. Eng. 36(8), 836-843 (1989).

77 De Ridder D, Plazier M, Kamerling N, Menovsky T, Vanneste S. Burst spinal cord stimulation for limb and back pain. World Neurosurg. doi:10.1016/j.wneu.2013.01.040 (2013) (Epub ahead of print). 